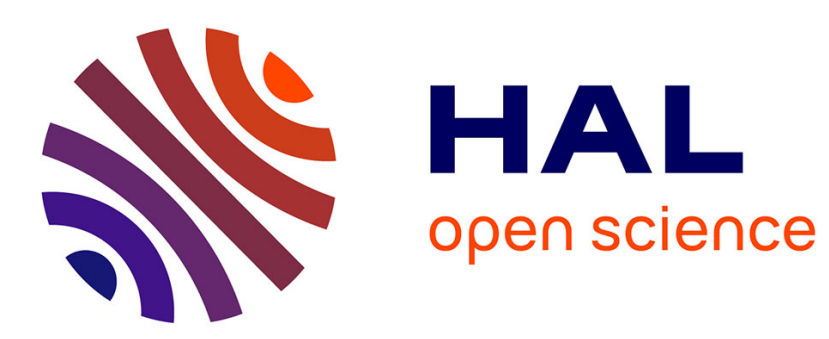

\title{
Fuzzy spatio-temporal relations analysis
}

Nadeem Salamat, El-Hadi Zahzah

\section{To cite this version:}

Nadeem Salamat, El-Hadi Zahzah. Fuzzy spatio-temporal relations analysis. 7th International Conference on Information Technology: New Generations ITNG 2010, Apr 2010, Las Vegas, Nevada, United States. pp.00 00. hal-00472793

\section{HAL Id: hal-00472793 https://hal.science/hal-00472793}

Submitted on 13 Apr 2010

HAL is a multi-disciplinary open access archive for the deposit and dissemination of scientific research documents, whether they are published or not. The documents may come from teaching and research institutions in France or abroad, or from public or private research centers.
L'archive ouverte pluridisciplinaire HAL, est destinée au dépôt et à la diffusion de documents scientifiques de niveau recherche, publiés ou non, émanant des établissements d'enseignement et de recherche français ou étrangers, des laboratoires publics ou privés. 


\section{Fuzzy spatio-temporal relations analysis}

\author{
Nadeem SALAMAT \\ MIA laboratory \\ University of La Rochelle, Avenue M. Crepeau \\ La Rochelle 17042, France \\ nadeem.salamat@univ-lr.fr
}

\author{
El-hadi ZAHZAH \\ MIA laboratory \\ University of La Rochelle, Avenue M. Crepeau \\ La Rochelle 17042, France \\ ezahzah@univ-lr.fr
}

\begin{abstract}
There are different families of Spatio-temporal relations such as same-place same-time, same-place differenttimes, for road networks like overtake, derive beside and many others. These relations describe the relative positions of objects in a spatial scene. In existing techniques, these relations are defined qualitatively. Due to imprecise knowledge information and compensation power to small errors, fuzzy methods are becoming more important.

In this paper, fuzzy spatio-temporal relations same-place differenttime and different-place different time are introduced. To define these relations, histograms of fuzzy Allen relations and fuzzy dissimilarity measure are used.
\end{abstract}

Keywords-Spatio-temporal relations, Relative position, Fuzzy Allen relations, Quantitative spatial relations, Fuzzy dissimilarity measure

\section{INTRODUCTION}

A lot of work has been done in developing the fuzzy spatial relations. These relations include the topological, directional and distance relations. Nowadays the most favorite topic for researchers community in this field is spatio-temporal relations. These relations are mostly depend on their applications domain. In this regard, different examples are considered. A spatial scene can be completely described by geometric objects along with their spatial relations such as topological, distance and directional relations. Mostly spatio temporal relations are studied in a separate domains and a variety of researchers such as M. Schneider [6] described the different spatio-temporal relations which involve topological changes. Nico Van de Weghe et al. [15], [11], [8] develop Qualitative Trajectory Calculus (QTC) which deals with moving objects and it always consider the disjoint objects. They used spatio-temporal changes to model the relations between moving objects on road networks. These spatio temporal changes are essential part of artificial intelligence, video content description. In [16] fuzzy directional relations are used ( Histogram of forces ) to describe the video contents. Movement or expansion of spatial objects can cause to change the topological, metric or both at the same time. Following are the examples of different moving objects, which shows that moving objects can change the shape, size and locations as a result in the scene the topological, metric or directional relations between the geometric objects are changed.

- A $\log$ close to a lake may go through a variety of topological relationships. When the water surface is too low, that there is a land between the water surface and $\log$. With increase in water level, the log will have immediate access to the water surface with continuous increase in water surface log first partially under water then completely under water at edge and finally it may become an island in lake. These relations are explained in figure 1 .

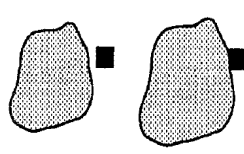

(a)

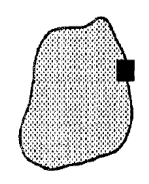

(c)

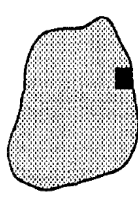

(d)

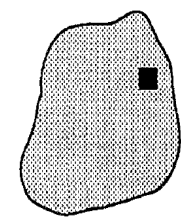

(c)
Fig. 1. Changes in topological relationship between lake and log. [2]

- A cat jumps over the wall, then both the objects don't change their size, only directional relation is changed at each instant of time. A pictorial scene looks like figure 2, where circular object is used to represent the cat position.

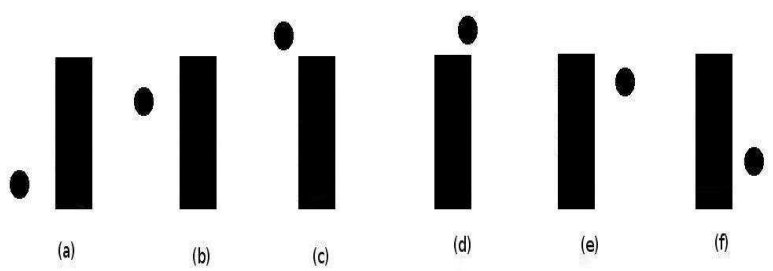

Fig. 2. View of cat jump over the wall

- A boat with in the lake continuously changes its position with respect to an observer standing out side the lake. For example first of all the boat was near the eastern edge of the lake, then it changes continuously its position towards north and finally it reaches near the north edge of lake. In such a case the boat changes its locations. In figure 3 boat is represented by a small rectangle and big polygon shape object represents the lake.

They move continuously and they change their positions to the neighborhood for this purpose the concept of gradual changes in topological relations was introduced. Models for changes of topological relationship are relevant to spatio-temporal 


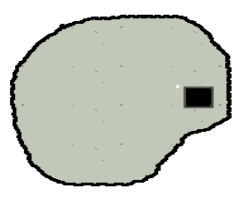

a

Fig. 3. A boat inside a lake continuously changes its position reasoning in geographic spaces, as they derive the most like configuration and allow for prediction about significant changes. Such predictions are important methods for reasoning in large scale space and geographic spaces [2] and point set topology is used to determine the topological relations and the topological distances are defined between the topological relations to observe that how much change has occurred. In this model object movements which causes changes in directional and distance information are not considered and same object pair is considered at two different times. This model to detect the gradual changes in spatial scene can be used in situation like explained in figure1, where dynamic spatial relations are considered and the major focus is on topological changes of the space. A similar approach is adopted by John Z. [3] and the qualitative directional relations are computed, trajectory of moving objects is represented by 8 directions and distance between these qualitative directional relations are defined to assess the gradual changes in directional relations. This model can be used to detect the spatial changes in directional relations where the situation like in figure 2 exists. This method is inspired by Max J. Egenhofer's method [2] of topological distances by considering the directional relations.

In situations like in figure 3, where the distance and directional information becomes invalid and the object does not change the topological relations Sung-Hye et al. [10] introduced the method of hierarchical spatial relations based on contiguity graph. This method depends upon the physical properties of objects not on the spatial properties. Another method in qualitative domain, is internal cardinal directions [14] which capture the position of an object inside the other object. This method is based on interval calculus and in this method the central tile or minimum bounding rectangle $(\mathrm{MBR})($ Also called Minimum Bounding Box (MBB)) for the 9-intersection method is divided into 4 or 9 or 13 subtiles. Qualitative methods can not detect small changes while the objects are overlapping, contained, contained_by or disjoint. More precisely the methods are qualitative not fuzzy. In defining the spatio- temporal relations rigid objects are considered they don't change or split during the movement. Moreover camera motions such as booming, tilting, panning and zooming are not considered.

This paper investigates that how an existing method of combined extraction of topological and directional relations [9], [18] can be used to detect the topological or metric changes between the two spatial objects. These changes can be used to define the spatio-temporal relations. In this paper section 2 covers the different aspects of a histogram of fuzzy Allen relations, in section 3 spatio-temporal relations are defined, section 4 covers the effect of spatial scene change and its measure. in section 5 different experiments are taken into account and section 6 concludes the paper.

\section{Histogram OF FUZZY AlLEN RELATIONS AND TERMINOLOGY USED}

In this section, the different terms used in this paper and the computation of histogram of fuzzy Allen relations are explained.

\section{A. Oriented lines, segments and longitudinal sections}

Let $A$ and $B$ be two spatial objects, $(v, \theta) \in R$, where $v$ is any real number and $\theta \in[-\pi, \pi] . \Delta_{\theta}(v)$ is an oriented line at orientation angle $\theta . A \cap \Delta_{\theta}(v)$ is the intersection of object $A$ and oriented line $\Delta_{\theta}(v)$. It is denoted by $A_{\theta}(v)$, called segment of object $A$ and its length is $x$. Similarly for object $B$ where $B \cap \Delta_{\theta}(v)=B_{\theta}(v)$ is segment and $z$ is its length. $y$ is the difference between the maximum value of $B \cap \Delta_{\theta}(v)$ and minimum of $A \cap \Delta_{\theta}(v)$ (for details [9]). In case of polygonal object approximations $(x, y, z)$ can be calculated from intersecting points of line and object boundary. If there exist more than one segment then it is called longitudinal section as in case of $A_{\theta}(v)$ in figure 4. In this paper all

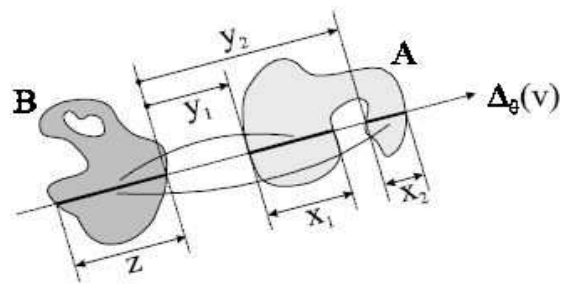

Fig. 4. Oriented line $\Delta_{\theta}(v)$, segment as in case of object $B$, longitudinal section as in case of object A.( Matsakis [9])

360 directions are considered with an angle increment of one degree and lines are drawn by $2 \mathrm{~d}$ Bresenham digital line algorithm. A polygonal object approximation is taken and lines passing through at least one vertex of the polygon are taken into account. segments are computed and all pairs of segments can be treated simultaneously. Fuzzy Allen relations are computed for each segment.

\section{B. ID Allen relations in space}

Allen in [1], introduced the well known 13 jointly exhaustive and pairwise disjoint (JEPD)interval relations based on temporal interval algebra. These relations are arranged as $A=\left\{<, m, o, s, f, d, e q, d_{i}, f_{i}, s_{i}, o_{i}, m_{i},>\right\}$. where $\{<, m, o, s, f, d\} \quad,\left(\left\{d_{i}, f_{i}, s_{i}, o_{i}, m_{i},>\right\}\right)$ are the relation before, meet, overlap, start, finish, during (resp the inverse relations of the cited ones). The relation $e q$ is the equality spatial relation. All the Allen relations in space are conceptually illustrated in figure 5 . These relations have a rich support for the topological and directional relations. 


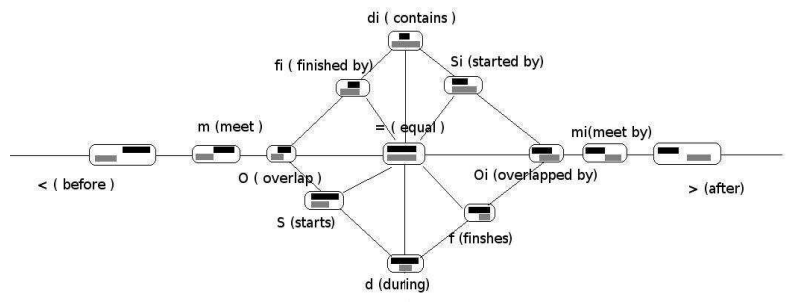

Fig. 5. Black segment represents the reference object and gray segment represents argument object

\section{Fuzzification of Allen relations}

In real applications, small errors in crisp values can change the entire result when gradual changes of topological relations occur over time. To cope with these problems fuzzification was introduced, it comprises the process of transforming crisp values into grades of membership for linguistic terms of fuzzy sets. Fuzzification process of Allen relations do not depend upon particular choice of fuzzy membership function, trapezoidal membership function is used due to flexibility in shape change. Let $r(I, J)$ is Allen relation between segments $I$ and $J$ where $I \in A$ (argument object) and $J \in B$ (reference object), $r^{\prime}$ is the distance between $r(I, J)$ and its conceptional neighborhood. We consider a fuzzy membership function $\mu: r^{\prime} \longrightarrow[0,1]$. Some of the fuzzy Allen relations defined by Matsakis [9] are:

- $f_{b}(I, J)=\mu_{(-\infty,-\infty,-b-3 a / 2,-b-a)}(y)$,

- $f_{a}(I, J)=\mu_{(0, a / 2, \infty, \infty)}(y)$

- $f_{m}(I, J)=\mu_{(-b-3 a / 2,-b-a,-b-a,-b-a / 2)}(y)$,

- $f_{m i}(I, J)=\mu_{(-a / 2,0,0, a / 2)}(y)$

- $f_{O}(I, J)=\mu_{(-b-a,-b-a / 2,-b-a / 2, b)}(y)$,

- $f_{O i}(I, J)=\mu_{(-a,-a / 2,-a / 2,0)}(y)$

- $f_{f}(I, J)=\min \left(\mu_{5}, \mu_{3}, \mu_{1}\right)$

- $f_{f i}(I, J)=\min \left(\mu_{6}, \mu_{4}, \mu_{2}\right)$

- $f_{s}(I, J)=\min \left(\mu_{6}, \mu_{4}, \mu_{1}\right)$

- $f_{s i}(I, J)=\min \left(\mu_{5}, \mu_{3}, \mu_{2}\right)$

- $f_{d}(I, J)=\min \left(\mu_{7}, \mu_{1}\right)$

- $f_{d i}(I, J)=\min \left(\mu_{7}, \mu_{2}\right)$

where $\mu_{1}=\mu_{(-\infty,-\infty, z / 2, z)}(x)$,

$\mu_{2}=\mu_{(z, 2 z,+\infty,+\infty)}(x)$,

$\mu_{3}=\mu_{(-3 a / 2,-a,-a,-a / 2)}(y)$,

$\mu_{4}=\mu_{(-\infty,-\infty,-b,-(b+a) / 2)}(y)$,

$\mu_{5}=\mu_{(-(b+a) / 2,-a,-a,+\infty)}(y)$,

$\mu_{6}=\mu_{-b-a / 2,-b,-b,-b+a / 2}(y)$,

$\mu_{7}=\mu_{(-b,-b+a / 2,-3 a / 2,-a)}(y), a=\min (x, z)$,

$b=\max (x, z), x$ is the length of longitudinal section of argument object $A$, and $z$ is the length of longitudinal section of reference object $B$. Most of relations are defined by one membership function and some of them by the minimum value of more than one membership functions like $d$ (during), $d_{i}$ (during_by), $f$ (finish), $f_{i}$ (finished_by). In fuzzy set theory, sum of all the relations is one, this gives the definition for fuzzy relation equal. Fuzzy Allen relations are not Jointly Exhaustive and Pairwise Disjoint (JEPD) because there exist at least two relations between two spatial objects.

\section{Treatment of longitudinal sections}

During the decomposition process of an object into segments, there can be multiple segments depending on object shape and boundary which is called longitudinal section. Different segments of a longitudinal section are at a certain distance and these distances might effect end results. Fuzzy Allen relations are computed for each segment then fuzzy operators are used for information fusion by considering that each Allen relation is a fuzzy set. Here fuzzy $O R$ operator $\left(\mu_{(O R)}(u)=\max \left(\mu_{(A)}(u), \mu_{(B)}(u)\right)\right)$ is used. For this operator, only one fuzzy value contributes for the resultant value which is maximum. In this case each Allen relation has a fuzzy grade objective is to accumulate the best available information. Suppose that longitudinal section of object $B$ has two segments such that $z=z_{1}+z_{2}$ where $z_{1}$ is the length of first segment and $z_{2}$ is the length of second segment and $z$ is length of longitudinal section. Let $\mu_{1}\left(y_{1}\right)$ defines the value of fuzzy Allen relations with the first segment and $\mu_{2}\left(y_{2}\right)$ represents value of fuzzy Allen relations with the second segment where $y_{1}$ and $y_{2}$ are the distances between object $A$ and two segments of $B$. Now fuzzy $O R$ operator is used to get consequent information obtained from two sets of fuzzy Allen relations.

\section{E. Fuzzy histogram of Allen relations and its normalization}

Histogram of fuzzy Allen relations represents the total area of subregions of $A$ and $B$ that are facing each other in given direction $\theta$ [9]. Area of subregions of object $A$ and

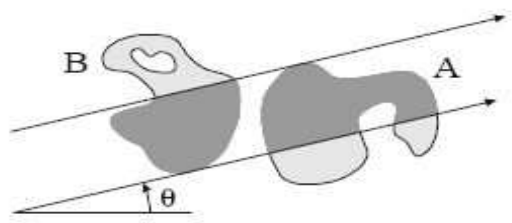

Fig. 6. Histogram of fuzzy Allen relation in direction $\theta$

$B$ represented by dark gray color in figure 6 represents a histogram of fuzzy Allen relation in given direction $\theta$. In case of polygonal object approximation which is considered by $\mathrm{N}$. Salamat and El Zahzah [18] to meet the problem of temporal complexity, fuzzy histogram of Allen relations can be written mathematically as:

$$
\int_{-\infty}^{+\infty}\left(\sum_{r \in A} F_{r}\left(q, A_{q}(v), B_{q}(v)\right) d v=(x+z) \sum_{k=1}^{n} r\left(I_{k}, J_{k}\right)\right.
$$

where $z$ is the area of reference object and $x$ is area of augmented object in direction $\theta, n$ is total number of segments treated and $r\left(I_{k}, J_{k}\right)$ is an Allen relation for segments $I_{k}, J_{k}$. Allen histograms can easily be normalized by dividing all Allen relations by the sum of all the Allen relations for every $\theta$ in the given direction. It is represented by $\left[F_{r}^{A B}(\theta)\right\rfloor$ where $r \in A .\left\lceil F_{r}^{A B}(\theta)\right\rfloor=\frac{F_{r}^{A B}(\theta)}{\sum_{\rho \in A} F_{\rho}^{A B}(\theta)}$. 


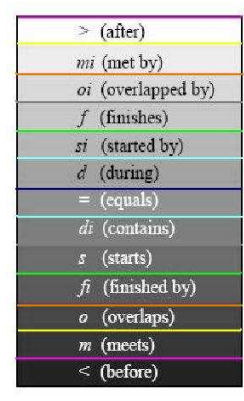

(a)

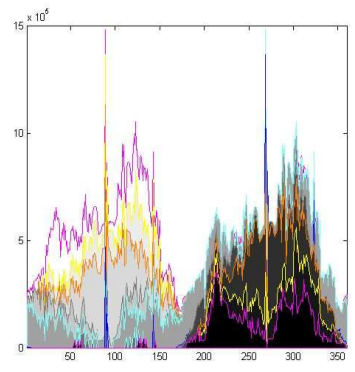

(c)

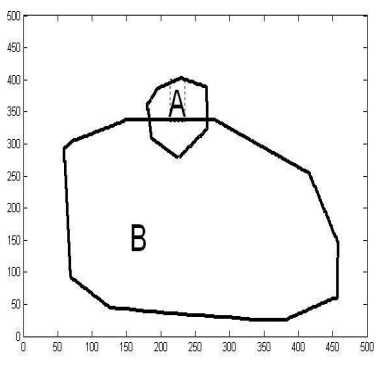

(b)

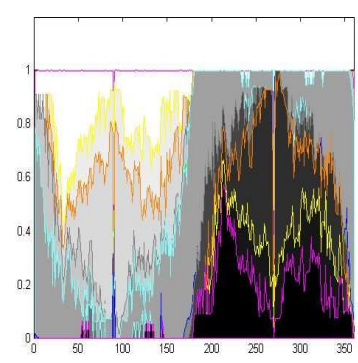

(d)
Fig. 7. (a) Histogram representation (b) Overlapping objects (c)Un normalized histogram of fuzzy Allen relations (d) Corresponding normalized histogram of fuzzy Allen relations

\section{SPATIO-TEMPORAL RELATIONS}

Thomas Bittner in [5] has introduced the some spatio temporal relations such as same-place same-time, sameplace different-time, different-place same-time, different-place different-time. Mathematically some of the relations have no back ground because it is almost impossible to express the spatio temporal relations same-place same-time. Logically these type of relations exist when one object $A$ causes object $B$ or there is a effect of $A$ on $B$ due to the presence of both object at same place at same time. In the same way the relation different-place same -time is also logical. This also has the same foundations that $A$ could not effect the object $B$ because both were at different places at the same time. Moreover these relations can hold in physical domain. Here our interest is to define the spatio-temporal relations same-place differenttime and different-place different-time in the spatial domains or in video analysis. if one object changes its position with respect to the other object in a scene at different time instants, then it is called that object $\mathrm{A}$ is at different place with respect to the object $B$ and if it doesn't change its relative position then it is called that object $A$ has the same-place at different times. An other important fact that the concept of same-place different times can also represent the circular movement of an object around that other object. For such a case object repeats its object location $2 \pi$ directions. Both of the relations are associated with a change in a spatial scene. In the next section, the occurrence of a spatial change and the method to measure the change is discussed. For change measure a dissimilarity measure is used and if at least one out of 13 dissimilarity measure is non zero then the spatio-temporal relation differentplace different-time exist otherwise the spatio-temporal relation same-place different time exists.

\section{Change Detection}

This section consist of two parts, first the effect of a change on a fuzzy histogram of Allen relations, second section describes how to measure these changes.

\section{A. Effect of change on fuzzy histogram of Allen relations}

To describe the relative position of object pair in an image, there exist three types of relations named as topological, directional and distance relations. These spatial relations are explained in figure 8 . When the relations are captured with

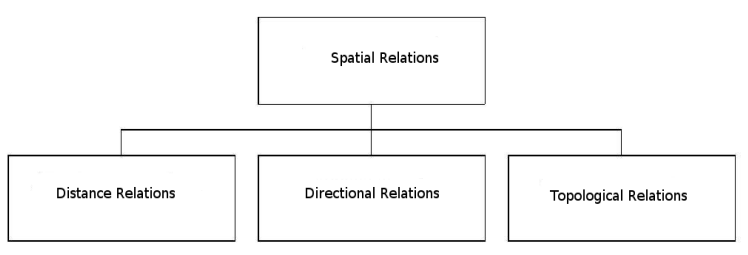

Fig. 8. Spatial Relations for a static image

the help of 1D Allen relations, the distance relations has the importance when the objects are disjoint. For the disjoint relations the distance relations can be estimated from the support of a fuzzy Allen histogram because directional relations are proportional to the visual rang of object [17]. This range is inversely proportional to the distance between the objects.i.e. Closer the distance between objects, larger will be the support for the fuzzy Allen relations histograms and vice versa. Thus the above relations can be described as

\section{(Fuzzy Allen Relations, Support of histograms, $T$ )}

where fuzzy Allan relations represents the thirteen fuzzy Allen relations and support of histograms represents the directions where histograms have non zero values and it is an interval between $[0,2 \pi]$ of a period $2 \pi$ and $T$ is time. To detect the change in a spatial scene, a snap shot scene model is considered and histograms of fuzzy Allen relations between same object pair can be compared at two different time instants.

Object under relative motion may undergo the changes in topological structure of scene or topological structure remains same but it changes the order or metric relations. Normalized fuzzy histograms are considered as fuzzy sets, where core and support of a fuzzy set depends directional and distance contents, if core and support is translated, then object changes only directional relation and if support shrinks or expands then object changes the distance relations and change detection can be considered as the inverse of assessing similarity between spatial scene, for detecting change in a spatial scene three types of relations, topological, directional and distance relations are compared. When the spatial relations are computed by the 
fuzzy histogram of Allen relations, the normalized histograms are considered as a fuzzy set whose support is inversely proportional to the distance for each topological relation.

\section{B. Change measure}

This change in the spatial scene can exist in three different ways, change in topological, directional and distance relations. These changes can effect the fuzzy Allen histograms in three ways (i) Emerges a new histogram or disappears an existing histogram,(ii) Changes the support of a histogram i.e. histogram support shrinks or expands (iii) Histogram support is translated. When an existing histogram disappears or new histogram appears it means object changes its topological relations. When the histograms expand or shrinks then the object is changing the distance information. To measure these changes different methods of similarity or dissimilarity can be used, as they are the inverse of each other. A similarity measure is used to access the degree of similarity of two images according to spatial relations between objects. Dissimilarity is the negation of similarity so dissimilarity could be defined as $\mu_{\text {Dissimilarity }}=\overline{\mu_{c}}=1-\mu_{c}$ and $0 \leq \overline{\mu_{c}} \leq 1$. Where $\overline{\mu_{c}}=0$ shows that there is no change in spatial scene and $\overline{\mu_{c}}=1$ shows that there is a complete topological or directional change in the spatial scene.

$$
\mu_{c}\left(h_{1}, h_{2}\right)=\frac{\sum_{\theta}\left(h_{1}(\theta) h_{2}(\theta)\right)}{\sqrt{\sum_{\theta}\left(h_{1}^{2}(\theta)\right.} \sqrt{\left.\sum_{\theta} h_{2}^{2}(\theta)\right)}}
$$

$\mu_{c}$ is normalized cross correlation for similarity measure. Normalized cross correlation is invariant to the camera zooming so due to this reason it is preferred to use this similarity measure. A vector or a similarity row or column matrix could be defined. If each element of this matrix is zero then there is no spatial change in the spatial scene and the spatio-temporal relation is defined as same-place different-time otherwise the relation will be different-place different-time.

\section{EXPERIMENTS}

For the experiment purposes, all the 13 fuzzy Allen histograms are calculated from angle measure $[0,360]$ with the increment of one degree and similarity between them. In the first example (figure 9(a) to 9(d)), we consider two frames of a well known image sequence of Y. Sheikh and M. Shah [7] where both objects are moving in opposite directions where the speed of the car is $h_{1}$ and speed of man is considered as $h_{2}$. If the one object say reference object $B$ (car) is considered at rest and the object $A$ is under motion, then net speed of object $A$ will be $h_{1}+h_{2}$. Here manual segmentation is taken, because here the problem is to detect the change in the scene not the segmentation. As at each frame the distance between the object increases, at the first analysis frame the fuzzy meet relation exists i.e. in terms of Allen relations fuzzy meet and meet_by relations exists along with certain directions. When the distance between the objects increase, the histogram for the relations meet and meet_by. Then the similarity between the two histograms are calculated. All those histograms which don't exist, the similarity or dissimilarity
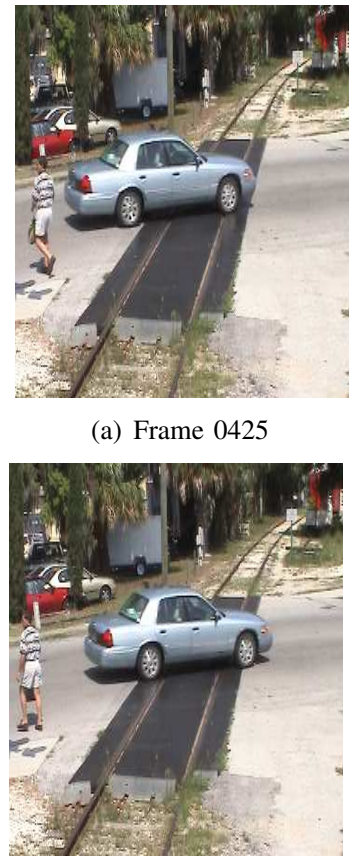

(c) Frame 0430 (a) Frame 0425 (b)

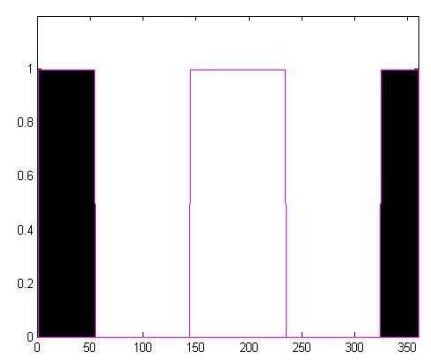

(d)

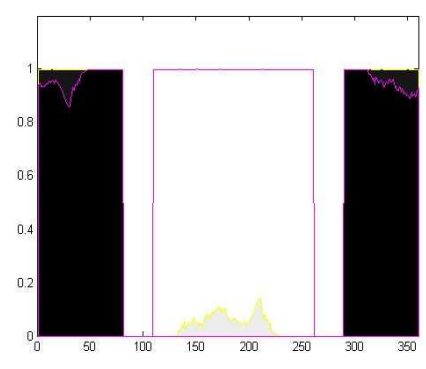

Fig. 9. Two frames of an image sequence of Y. Sheikh and M. Shah [7] and their spatial relations

between them is considered to be zero. Dissimilarity matrix will be $(0.3,1,0,0,0,0,0,0,0,0,0,1,0.3)$. This shows that the spatio-temporal relation is different-place different time. In second example (For reference figure 10(a) to 10(d)) In

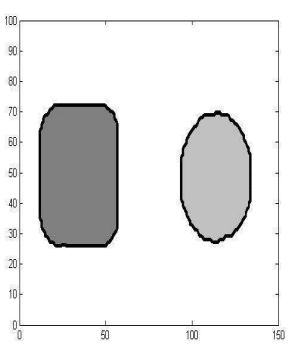

(a)

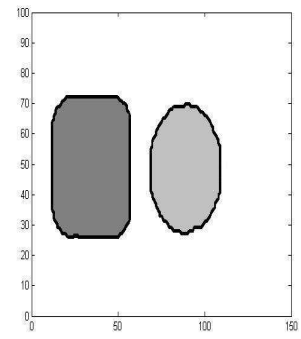

(c)

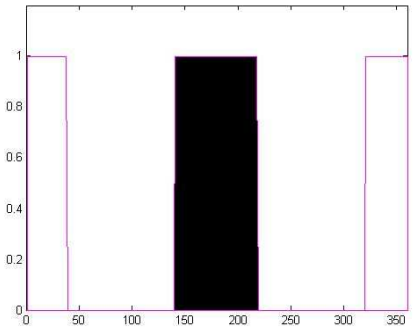

(b)

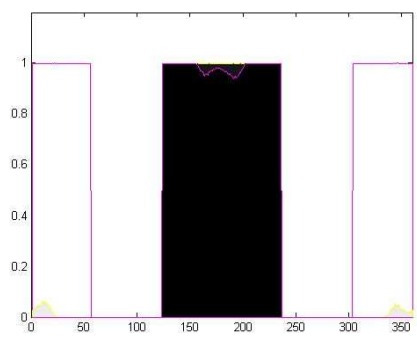

(d)
Fig. 10. (a) and (c) are images where in (c) object A (light gray object) moves towards object $\mathrm{B},(\mathrm{b}),(\mathrm{d})$ are their histogram representations.

this example two trail objects are considered for experimental purposes, rectangular object $B$ represented by dark gray color, 
and circular object $A$ is represented by the light gray color. In fact, the example represents the inverse phenomenon to the example 1. At initial stage the objects are at a certain distance, they have only disjoint topological relation, when the distance between them decreases to a certain extent, emerges a new histogram for the fuzzy meet relation. Although the objects don't meet but fuzzification is object size dependant. As a result, whole topological structure is changed and a new fuzzy topological relations exists in certain directions. Dissimilarity matrix will be $(0.2,1,0,0,0,0,0,0,0,0,0,1,0.2)$. This shows that the spatio-temporal relation is different-place different time.

In third example (figure 11(a) to 11(d)) when the object $A$ (light gray object )is inside the object $B$ (dark gray object), fuzzy Allen histograms are calculated there exist number of histograms due to fuzzification. In the second case object $A$ slightly changes its position while remaining inside the object $B$, for experimental purpose object $A$ is translated by $(-10,20)$, this results change in the fuzzy histograms. In this example qualitative directional and distance relations don't exist, and there is no qualitative change in spatial scene. But in fact there is a change in spatial scene, that object $A$ has changed its location. Dissimilarity matrix will

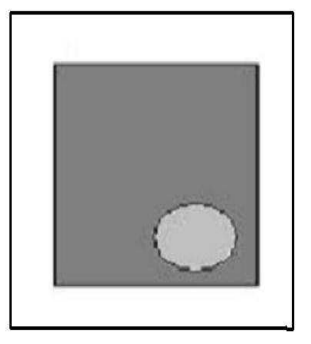

(a)

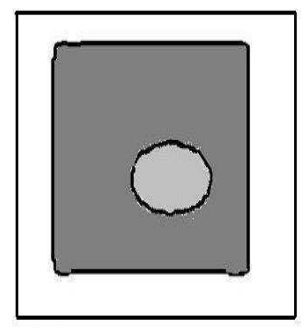

(c)

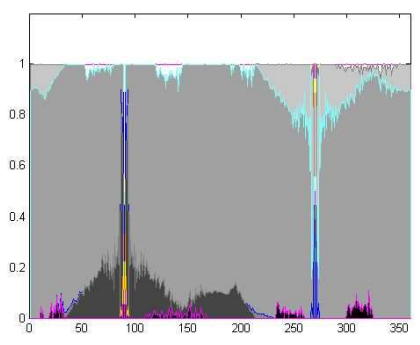

(b)

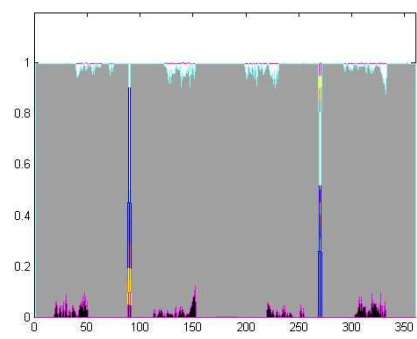

(d)
Fig. 11. (a) and (c) are images where in (c) object A moves towards the center of object B (b) and (d) are their histogram representations.

be $(0.4,0,0,0.9,0,0,0,0,0,0.9,0,0,0.4)$. This shows that the spatio-temporal relation is different-place different time. All the above cited examples are representing the spatio-temporal relation different-place different-time because each time objects are taken under motion.

\section{CONCLUSION}

Fuzzy techniques are becoming important day by day due to handling the vagueness and uncertainty in every field of image processing, computer vision and database management, defining the fuzzy spatio temporal relations is one of them. In this paper we have proposed a fuzzy method to define the spatio-temporal relations belonging to the family of sameplace different-time and different-place different-time. This method is based on our previous work [19], where we have defined a fuzzy method for detecting a small change in video scene analysis. The fuzzy Allen relations are used for the model and fuzzy dissimilarity measure is used to compare the dissimilarity for fuzzy Allen relations histograms.

\section{REFERENCES}

[1] Allen JF, Maintaining Knowledge about Temporal Intervals, Communications of the ACM 26(11) (1983), 832-843.

[2] Max J. Egenhofer and Khaled K. Al-Taha, Reasoning about Gradual Changes of Topological Relationships, Proceedings of the International Conference On GIS - From Space to Territory (London, UK), SpringerVerlag, 1992, pp. 196-219.

[3] John Z. Li, M. Tamer, Duane Szafron, and Sw Gbt Ddi, Modeling of Moving Objects in a Video Database, Proceedings of IEEE International Conference on Multimedia Computing and Systems, 1997, pp. 336-343.

[4] C. Shim and J. Chang, Content-based retrieval using trajectories of moving objects in video databases, Database Systems for Advanced Applications, International Conference on 0 (2001), 0168.

[5] Thomas Bittner, Qualitative spatio-temporal relations, Department of Computer Science, Northwestern University (2001), 1-13.

[6] Ralf H. Güting and Markus Schneider, Moving objects databases, Morgan Kaufmann Publishers, 2005.

[7] Yaser Sheikh and Mubarak Shah, Bayesian modeling of dynamic scenes for object detection, IEEE Transactions on Pattern Analysis and Machine Intelligence 27 (2005), no. 11, 1778-1792.

[8] Nico Van de Weghea Anthony G. Cohnb Philippe De Maeyera, Frank Witlox, Representing Moving Objects in Computer-based Expert Systems: The Overtake Event Example, Expert Systems with Applications 29 (November 2005), no. 4, 977-983.

[9] P.Matsakis and Dennis Nikitenko, Combined Extraction of Directional and Topological Relationship Information from 2D Concave Objects, in fuzzy modeling with spatial informations for geographic problems, Springer-Verlag Publications, pp. 15-40, New York, 2005.

[10] Sung-Hye Choe, Jong-Hee Park, and Sung-Soo Pyo, Hierarchical spatial relation based on a contiguity graph: Research articles, Int. J. Intell. Syst. 20 (2005), no. 9, 867-892.

[11] Nico Van de Weghe, Anthony G. Cohn, Guy De Tre, and Philippe De Maeyer, A qualitative trajectory calculus as a basis for representing moving objects in geographical information systems, Control and Cybernetics 35 (2006), no. 1, 97-119.

[12] Valerie De Witte1 Tom Mlange1 M.Nachtegael, S.Schulte and Etienne E. Kerre1, Image Similarity From Fuzzy Sets to Color Image Applications, Series Lecture Notes in Computer Science, vol. 4781/2007, pp. 26-37, Springer Berlin.

[13] Colin Robertson, Trisalyn Nelson, Barry Boots, and Michael A. Wulder, Stamp: spatial-temporal analysis of moving polygons, Journal of Geographical Systems 9 (2007), no. 3, 207-227.

[14] X Wang Y Zhang X Jin Y Liu, X Liu, Qualitative Spatial Reasoning About Internal Cardinal Direction Relations, Advances in SpatioTemporal Analysis, CRC.

[15] Matthias Delafontaine, Nico Van de Weghe, Peter Bogaert, and Philippe De Maeyer, Qualitative Relations Between Moving Objects in a Network Changing its Topological Relations, Inf. Sci. 178 (2008), no. 8, 1997-2006.

[16] Archana M. Rajurkar, R.C. Joshi, and Santanu Chaudhary, Video content description using fuzzy spatio-temporal relations, Hawaii International Conference on System Sciences 0 (2008), 139.

[17] Min Deng.Zalim li, A Statistical Model for Directional Relations Between Spatial Objects, GeoInformatica 12(2) (2008), 193-217.

[18] Nadeem Salamat and El hadi Zahzah, Spatial relations analysis by using fuzzy operators., ICCS (2), Lecture Notes in Computer Science, vol. 5545, Springer, 2009, pp. 395-404.

[19] Nadeem Salamat and El hadi Zahzah, Fuzzy change detection in a spatial scene. accepted for publication in proceedings of geoprocessing 2010. 